\title{
Is the Horizontal-Vertical Illusion Mainly a By-Product of Petter's Rule?
}

\author{
Alessandra Pecunioso ${ }^{1, *}$ and Christian Agrillo 1,2 (D) \\ 1 Department of General Psychology, University of Padova, 35131 Padova, Italy \\ 2 Padua Neuroscience Center, University of Padova, 35131 Padova, Italy; christian.agrillo@unipd.it \\ * Correspondence: alessandra.pecunioso@studenti.unipd.it; Tel.: +39-0498276931
}

Received: 6 November 2019; Accepted: 17 December 2019; Published: 18 December 2019

\begin{abstract}
The horizontal-vertical (HV) illusion is a classical example of an asymmetrical perception of size in the vertical and horizontal axes, also known as 'anisotropy of the perceived space'. Several authors argued that the horizontally-oriented ellipse of the binocular visual field might play an important role in the emergence of this illusion. Alternatively, a length bisection bias and size-constancy mechanisms have been advocated to account for the asymmetrical perception in the two dimensions. To investigate this phenomenon, participants are commonly required to estimate the length of two separate lines, one vertical and one horizontal, often arranged in an inverted-T pattern. Here we suggest that this type of stimulus may introduce physical and subjective biases that prevent a fine investigation. In particular, we believe that Petter's rule, that applies to two-dimensional patterns formed by two overlapping surfaces, may play a critical role that will not support an interpretation based on the shape of the binocular visual field nor a length bisection bias.
\end{abstract}

Keywords: visual illusions; Petter's law; inappropriate size-scaling; Symmetry; perception; anitrotropy

\section{Introduction}

The horizontal-vertical (HV) illusion is a well-known size illusion in which two identical objects appear to be different when presented on the horizontal or vertical plane. Typically, the HV illusion appears as an inverted T, where the apparent length of the vertical line is estimated to be greater than the horizontal one. To date, most researchers agree that this perceptual bias is related to the peculiarity of our visual fields. The binocular visual field is a horizontally-oriented ellipse, with a horizontal-to-vertical aspect ratio of 1.53 compared to the ratio of 1.23 in the monocular field [1,2]. The ends of the vertical line are closer to the boundary of the visual field, leading to an assimilation of its length to the boundary edges of the visual field. In contrast, the ends of the horizontal one are far from the boundary of the visual field, a fact that would elicit a contrast effect. As a result of both effects, the vertical line would appear longer than the horizontal one [2-5].

Another explanation advanced in the literature deals with the presence of a bisected line: If a line is bisected in two parts, it often appears shorter than if it were not interrupted, a perceptual effect called the 'length bisection bias' [6,7]. This bias seems to play a role in the HV illusion, as it was found that we are more susceptible to the HV illusion in the ' $\mathrm{T}$ ' version (magnitude of the effect: approx. $16-20 \%$ ) than in the ' $L$ ' version, where no line is bisected (magnitude of the effect: approx. 3-6\% [4,7]). However, the fact that we perceive also the vertical line as longer than the horizontal in the ' $L$ ' version clearly indicates that the length bisection bias per se cannot explain the different perceptions that we have regarding the vertical and horizontal axes.

Other authors invoke the 'inappropriate size-scaling hypothesis' to explain the HV illusion [8-10]. According to this hypothesis, often advocated to explain different geometrical illusions (e.g., Müller-Lyer 
and Ponzo illusion [9]), the vertical line would be perceived as receding in the distance. The horizontal line, instead, would be seen to lie on the same plane. Because of the size-constancy mechanisms commonly involved to compensate for the decreasing retinal size of an item with increasing distance, the vertical line would appear longer. This hypothesis has been recently criticized by Wolfe et al. [11] who suggested that depth cues associated to the illusory stimuli cannot entirely account for observed length perception distortions in the vertical and horizontal space.

The existence of an anisotropy of the perceived space in the horizontal and vertical axes has been widely demonstrated in studies requiring participants to estimate the length of two separate lines, one vertical and one horizontal (e.g., a $\mathrm{T}$ or $\mathrm{L}$ version with no spatial continuity between the two lines [6,12]). However, in most of the cases [13-16], the anisotropy of the perceived space is studied by presenting participants with the $\mathrm{T}$ shape, where the horizontal and vertical lines are shown in spatial continuity (Figure 1). We suggest here that this type of stimulus may introduce physical/perceptual biases that are not related to the assimilation/contrast effects associated with our binocular visual fields or to the length bisection bias.

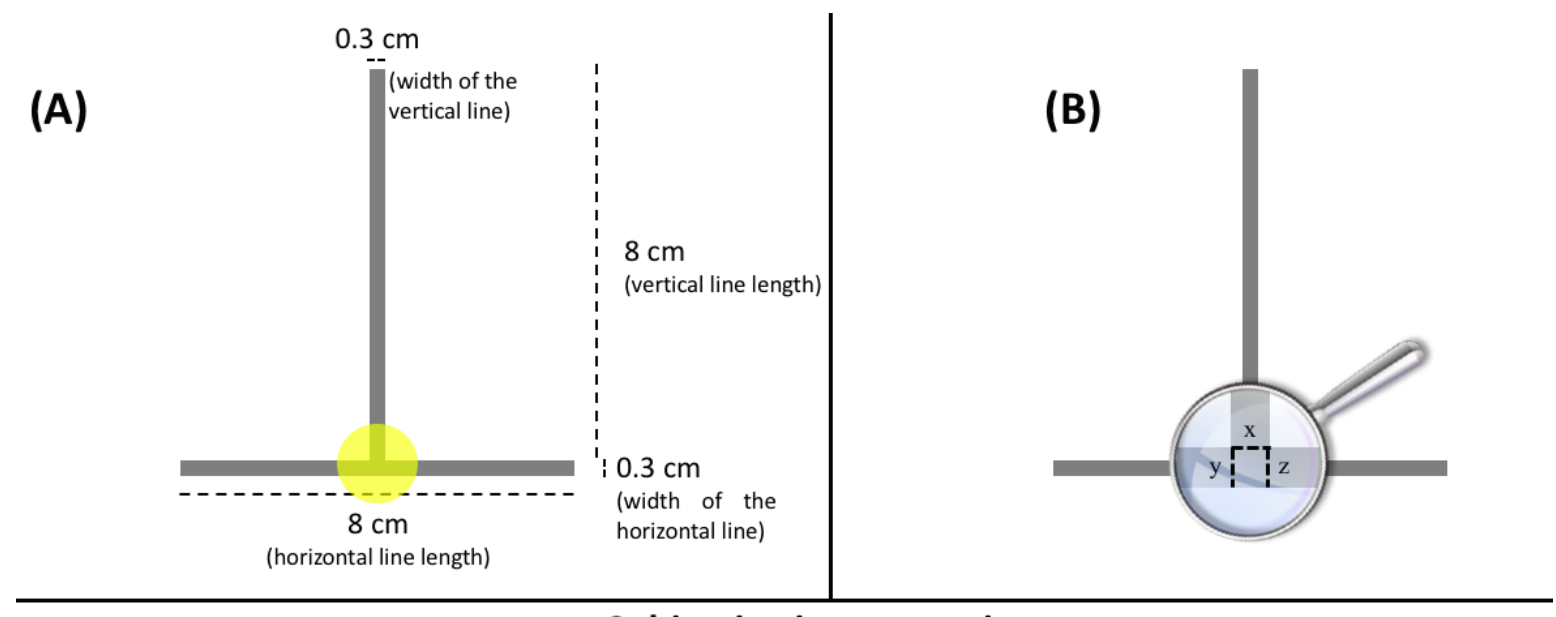

\section{(C)}

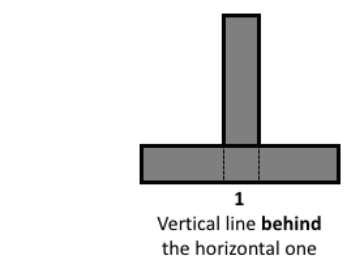

\section{Subjective interpretations}

\section{)}

Figure 1. (A) While studying the perception of the HV illusion, researchers should take into consideration two potential scenarios that might occur with the inverted T-pattern: First, if the vertical line is in contact with the horizontal line, without sharing a common area, a physical bias may affect the results of the study, as the length of the vertical line is objectively longer $(8+0.3 \mathrm{~cm}$ vs. $8 \mathrm{~cm}$ in the horizontal space). The yellow circle identifies the common area $(0.3 \times 0.3 \mathrm{~cm})$ between the horizontal and vertical lines that determines the bias. No physical bias occurs if the vertical and horizontal lines share a common area. (B) Petter's rule: The surface with the shorter contours in the common area has a greater chance of appearing in front of the other (in this case, $x<(y+z)$ ). Hence, we are supposed to perceive the vertical line behind the horizontal one. Petter's rule supports the inappropriate size-scaling hypothesis, according to which the vertical line appears to be farther away and is perceptually enlarged. (C) Three potential interpretations of this ambiguous pattern: (1) The vertical line appears behind the horizontal line, (2) lies on the horizontal one or (3) appears in front of the horizontal one. This latter interpretation is poorly supported by Petter's rule.

When researchers present vertical and horizontal lines in spatial contiguity, there are two different ways to represent the visual array: (a) the vertical line is in contact with the horizontal line, with 
no common area between the two lines; (b) the vertical line ends within the horizontal line, with a common area shared by the two lines. Here we will present two gross lines to make our argumentation as clear as possible (Figure 1). We are aware that authors use thinner lines in their studies. However, the use of thinner lines is likely to reduce but not eliminate the problems illustrated below.

\section{The Vertical and Horizontal Line Do Not Share a Common Area}

This scenario introduces a physical bias that might explain the different estimation between the two lines without invoking any illusory effect: The grey area presented in the vertical space is physically larger than that presented in the horizontal space (Figure 1A). For instance, if the two lines are $8 \mathrm{~cm}$ long and $0.3 \mathrm{~cm}$ wide, the grey area in the vertical space corresponds to $8.3 \times 0.3 \mathrm{~cm}^{2}$ (not $8 \times 0.3 \mathrm{~cm}^{2}$, as in the horizontal one). This represents a critical issue for a stimulus that is supposed to investigate a visual illusion based on identical stimulation in the two spatial dimensions.

It might be argued that the above-exposed scenario is counterintuitive in terms of subjective perception of this physical stimulation, because it contradicts the well-known Petter's rule [17]. This rule commonly applies to two-dimensional patterns formed by two overlapping surfaces that may alternately appear in front of one another. It states that the surface with the shorter contours, in the area in which the surfaces appear to be superimposed, has a greater chance of appearing in front of the other surface [18]. Petter's rule is robust and has been reported with several types of ambiguous patterns [18-21]. In the case of the T pattern, the subjective contour length necessary to perceive the horizontal line in front the vertical one (' $x$ ', Figure 1B) is shorter than the sum of the subjective contours necessary to perceive the vertical line in front of the horizontal one (' $y+z^{\prime}$, Figure 1B).

Provided that researchers cannot take for granted that subjective contours would compensate the physical bias presented with this type of stimulus, a potential bias might also occur in the case of Petter's rule acting on participants' perception. If we assume that the horizontal line is perceived in front of the vertical line (Figure 1(C1)), in agreement with Petter's rule, we would incur two concomitant biases: a physical bias (the area of grey in the vertical axis is larger) and a depth bias that is not related to either the anisotropy of the visual field or the bisection bias. The vertical line would appear longer because it is perceived as farther, supporting the inappropriate size-scaling hypothesis. On the contrary, if we suppose that participants perceive the vertical line as lying on the same surface as the horizontal line (Figure 1(C2)), the physical bias would still represent a critical confounder to drawing any conclusions on illusory phenomena. The criticism for the condition illustrated in Figure 1(C3) (vertical line appearing in front of the horizontal line) is always related to the physical bias. In addition, the mental representation of a vertical line in front of the horizontal line clearly violates Petter's rule, making this scenario less probable to occur.

Therefore, presenting a vertical line that is supposed to physically lie on a same-sized horizontal one is far from exempt from physical or subjective biases.

\section{The Vertical and Horizontal Lines Share a Common Area}

The other scenario consists of a stimulus in which the bottom of the vertical line is also part of the horizontal line. In this case, the lengths of the two stimuli are identical in terms of physical stimulation (e.g., $8 \mathrm{~cm}$ ), a condition that theoretically represents the ideal condition for studying the HV illusion (Figure 1A). That said, this argument works only if we assume that participants perceive the vertical line at the same distance as the horizontal one. Furthermore, it works if participants include the shared area $(0.3 \times 0.3 \mathrm{~cm})$ in both the vertical and horizontal lines, with the paradoxical condition according to which two objects simultaneously occupy the same portion of space.

Assuming that Petter's rule works in its classical direction (that is, the horizontal line perceived in front of the other, Figure 1(C1)), participants may provide different length estimations of the two lines for reasons that are not related to the anisotropy of the perceived space or the bisection bias. Again, the vertical line would appear to end behind the horizontal line, appearing to be farther and 
presumably longer than the horizontal one. The inappropriate size-scaling hypothesis would be the most likely explanation.

Another case is represented by the condition in which the vertical line would appear as lying on the horizontal one (Figure 1(C2)). As said above, the vertical line would appear shorter than the horizontal one, precisely $8-0.3=7.7 \mathrm{~cm}$, compared to $8 \mathrm{~cm}$ of the horizontal line. This would lead to an underestimation of the vertical line. The possibility exists that this initial underestimation would be compensated by the concomitant presence of the anisotropy of the perceived space and the length bisection bias, leading to the illusory effect commonly described in the HV illusion.

Lastly, if Petter's rule is violated (Figure 1(C3)), participants might perceive the vertical line in front of the horizontal one. If so, because the two lines occupy the same retinal size, the horizontal one should appear longer based on the inappropriate size-scaling hypothesis (again, a condition rarely found in the literature).

Therefore, presenting two lines that share a common surface appears correct in terms of physical stimulation but introduces different perceptual biases, of which researchers should be aware.

\section{Discussion}

In this work, we did not question the existence of anisotropy of the perceived space [1,2], nor did we question the solidity of the length bisection bias [4,7]. Instead, we sought to highlight the difficulty of drawing firm conclusions with the visual pattern most commonly used in this field. As argued here, the presentation of the T shape with spatial continuity between the two lines introduces physical and perceptual biases-the latter derived by the application of Petter's rule-that speak in favour of the inappropriate size-scaling hypothesis in most of the scenarios. As far as we are aware, no study has ever directly connected Petter's rule with the perceptual mechanisms underlying the HV illusion.

As stated in Introduction, researchers in this field commonly use thinner lines in their studies, thus reducing the problems here illustrated. Thinner lines, however, do not eliminate the problems, leaving open the possibility that hypotheses other than those related to the binocular visual field or the bisection bias may explain the results obtained when a vertical and horizontal line are in contact. More in detail, one may argue that participants would hardly complete amodally with the ambiguous information in the HV illusion, because the common surface between the two lines is very small compared to the rest of the visual array. However, amodal completion in a perceptual process occurs automatically in a very limited amount of time. Above all, it was demonstrated that the HV illusion also occurs when participants are simply required to imagine the two lines $[16,22]$, showing that it is the mental representation of the stimuli, rather than the physical stimulation per se, that seems to count more for the emergence of this size illusion. Provided that it is hard to simultaneously represent two objects in the same portion of space and at the same distance from the observer, there is a concrete possibility that participants represent the two lines at different distances, with Petter's rule guiding them in establishing which line is behind the other.

We believe that researchers must be cautious in drawing conclusions on the perceptual mechanisms underlying the different length estimations in the vertical and horizontal space when dealing with this type of visual pattern. Adopting different-coloured lines (e.g., a red vertical line lying on a black horizontal line) would make the pattern less ambiguous because it would eliminate most of these problems. The two lines would be equally long in terms of physical stimulation, and the vertical line would be perceived above the horizontal one. Still, it would not exclude the possibility that, due to Petter's rule, the vertical one would be perceived as being behind the horizontal one. We are aware that here we have only presented our personal viewpoint without providing data in support of our argumentations. However, even in the absence of empirical data, it must be noticed that lines arranged as those described in Section 2 present an intrinsic confound due to the different lengths of the two lines. In addition, we remarked that the application of Petter's rule, commonly accepted as an important mechanism to establish which surface is in front of another [18-21], cannot be ignored for both Sections 2 and 3 when searching for the exact perceptual mechanism underlying the misperception 
of size with the inverted T-pattern. We call for a wider discussion of these issues in the scientific community: More effort is required to study quantitatively the effect of lines' overlap (see [23] for a recent investigation about). Neglecting biases that might significantly alter the internal representation of the stimuli commonly used in the field (e.g., giving the impression of one line being above the other) could lead to understanding only half of the story.

Author Contributions: Both authors contributed equally to this work. All authors have read and agreed to the published version of the manuscript.

Funding: This research was funded by PRIN 2015 (grant number 2015FFATB7) from “Ministero dell'Istruzione, Università e Ricerca" (MIUR, Italy) and by STARS@unipd (acronym: ANIM_ILLUS) from the University of Padova (Italy) to C. Agrillo.

Conflicts of Interest: The authors declare no conflict of interest.

\section{References}

1. Harrington, D.O. The visual fields: A textbook and atlas of clinical perimetry. In Anatomy of Visual Pathway, 5th ed.; Mosby: St. Louis, MO, USA, 1981.

2. Künnapas, T.M. An analysis of the "vertical-horizontal illusion". J. Exp. Psychol. 1955, 49, 134. [CrossRef] [PubMed]

3. Künnapas, T.M. The vertical-horizontal illusion and the visual field. J. Exp. Psychol. 1957, 53, 405. [CrossRef] [PubMed]

4. Avery, G.C.; Day, R.H. Basis of the horizontal-vertical illusion. J. Exp. Psychol. 1969, 81, 376. [CrossRef] [PubMed]

5. Prinzmetal, W.; Gettleman, L. Vertical-horizontal illusion: One eye is better than two. Percept. Psychophys. 1993, 53, 81-88. [CrossRef]

6. Finger, F.W.; Spelt, D.K. The illustration of the horizontal-vertical illusion. J. Exp. Psychol. 1947, $37,243$. [CrossRef] [PubMed]

7. Mamassian, P.; de Montalembert, M. A simple model of the vertical-horizontal illusion. Vis. Res. 2010, 50, 956-962. [CrossRef] [PubMed]

8. Girgus, J.S.; Coren, S. Depth cues and constancy scaling in the horizontal-vertical illusion: The bisection error. Can. J. Psychol. 1975, 29, 59. [CrossRef]

9. Gregory, R.L. Distortion of visual space as inappropriate constancy scaling. Nature 1963, 199, 678-680. [CrossRef]

10. Gregory, R.L. Knowledge in perception and illusion. Philos. Trans. R. Soc. Lond. B 1997, 352, 1121-1127. [CrossRef]

11. Wolfe, U.; Maloney, L.T.; Tam, M. Distortions of perceived length in the frontoparallel plane: Tests of perspective theories. Percept. Psychophys. 2005, 67, 969-979. [CrossRef]

12. Cai, Y.; Wang, C.; Song, C.; Li, Z. Connectedness underlies the underestimation of the horizontal vertical illusion in L-shaped configurations. Atten. Percept. Psychophys. 2017, 79, 1217-1226. [CrossRef] [PubMed]

13. Charras, P.; Lupiáñez, J. Length perception of horizontal and vertical bisected lines. Psychol. Res. 2010, 74, 196-206. [CrossRef] [PubMed]

14. Gardner, R.W.; Long, R.I. Errors of the standard and illusion effects with the inverted-T. Percept. Mot. Skills 1960, 10, 47-54. [CrossRef]

15. Marma, V.; Bulatov, A.; Bulatova, N. Psychophysical study of vertical-horizontal illusion. Biomed. Eng. 2016, 19, 70-73.

16. Blanuša, J.; Zdravković, S. Horizontal-vertical illusion in mental imagery: Quantitative evidence. Front. Hum. Neurosci. 2015, 9, 33. [CrossRef] [PubMed]

17. Petter, G. Nuove ricerche sperimentali sulla totalizzazione percettiva. Riv. Psicol. 1956, 50, $213-217$.

18. Masin, S.C. Petter's effect in patterns formed by outlined surfaces. Percept. Mot. Skills 2000, 90, 595-600. [CrossRef]

19. Masin, S.C. Test of Petter's rule for perceived surface stratification. Perception 1999, 28, 1147-1154. [CrossRef]

20. Singh, M.; Hoffman, D.D.; Albert, M.K. Contour completion and relative depth: Petter's rule and support ratio. Psychol. Sci. 1999, 10, 423-428. [CrossRef] 
21. Kitaoka, A.; Gyoba, J.; Sakurai, K. The visual phantom illusion: A perceptual product of surface completion depending on brightness and contrast. Progr. Brain Res. 2006, 154, 247-262.

22. Wallace, B. Creation of the horizontal-vertical illusion through imagery. Bull. Psychon. Soc. 1984, $22,9-11$. [CrossRef]

23. Mikellidou, K.; Thompson, P. The vertical-horizontal illusion: Assessing the contributions of anisotropy, abutting, and crossing to the misperception of simple line stimuli. J. Vis. 2013, 13, 7. [CrossRef] [PubMed]

(C) 2019 by the authors. Licensee MDPI, Basel, Switzerland. This article is an open access article distributed under the terms and conditions of the Creative Commons Attribution (CC BY) license (http://creativecommons.org/licenses/by/4.0/). 